\title{
A simple proof of Linas's theorem on Riemann zeta function
}

\author{
Jun Ikeda ${ }^{1}$, Junsei Kochiya ${ }^{2}$ and Takato $\mathbf{U i}^{3}$ \\ ${ }^{1}$ Kaijo School, Shinjuku, Tokyo, Japan \\ e-mail: jun.ikeda0121@gmail.com \\ ${ }^{2}$ Kaijo School, Shinjuku, Tokyo, Japan \\ e-mail: 0125 . junsei@gmail.com \\ ${ }^{3}$ Kaijo School, Shinjuku, Tokyo, Japan \\ e-mail: uitakato@gmail.com
}

Received: 22 March 2021

Revised: 24 July 2021

Accepted: 1 November 2021

\begin{abstract}
Linas Vepštas gives rapidly converging infinite representatives for values of Riemann zeta function at $(4 m-1)$, where $m$ is a natural number. In this paper, we give a new simple proof. Also, we obtain two equation of values of Bernoulli numbers' generating function by applying a corollary given in this paper.
\end{abstract}

Keywords: Analysis, Riemann zeta function, Fourier series, Hyperbolic function.

2020 Mathematics Subject Classification: $11 \mathrm{M} 06$.

\section{Introduction}

Linas Vepštas [2] gave the following rapidly converging infinite representations for values of $\zeta(4 m-1)$ using polylogarithm, where $m$ is a natural number and $B_{k}$ is the $k^{\prime}$ th Bernoulli number.

Theorem 1.1 (Linas's theorem).

$$
\zeta(4 m-1)=-2 \sum_{n=1}^{\infty} \frac{1}{n^{4 m-1}\left(e^{2 \pi n}-1\right)}-\frac{1}{2}(2 \pi)^{4 m-1} \sum_{j=0}^{2 m}(-1)^{j} \frac{B_{2 j}}{(2 j) !} \frac{B_{4 m-2 j}}{(4 m-2 j) !} .
$$

In this paper, we give a simple proof of it, using Fourier series of $\cosh (x)$. 


\section{Preliminaries}

Lemma 2.1 (cf. [1, p. 506]).

$$
L \operatorname{coth}(L)=1+2 \sum_{n=1}^{\infty} \frac{1}{\left(\frac{\pi}{L} n\right)^{2}+1} .
$$

Proof. Using the Fourier series expansion method from $-L$ to $L$, where $L$ is a positive real number, $\cosh (x)$ is expressed as following

$$
\cosh (x)=\frac{\sinh (L)}{L}+\sum_{n=1}^{\infty} \frac{2}{L} \frac{(-1)^{n}}{\left(\frac{\pi}{L} n\right)^{2}+1} \sinh (L) \cos \left(\frac{\pi}{L} n x\right) .
$$

Substituting $L$ for $x$, we obtained the following equation.

$$
\cosh (L)=\frac{\sinh (L)}{L}+\frac{2}{L} \sinh (L) \sum_{n=1}^{\infty} \frac{(-1)^{n}}{\left(\frac{\pi}{L} n\right)^{2}+1}(-1)^{n} .
$$

\section{Corollary 2.1.1.}

$$
\sum_{k=1}^{\infty} \frac{\operatorname{coth}(\pi k)}{k^{4 m-1}}=\frac{1}{\pi} \sum_{k=1}^{\infty} \frac{1}{k^{4 m}}+\frac{2}{\pi} \sum_{k=1}^{\infty} \sum_{n=1}^{\infty} \frac{1}{k^{4 m-2} n^{2}+k^{4 m}} .
$$

Lemma 2.2.

$$
\sum_{k=1}^{\infty} \sum_{n=1}^{\infty} \frac{1}{k^{4 m}+k^{4 m-2} n^{2}}
$$

This series converges, where $m$ is a positive integer.

Proof. From Corollary 2.1.1, the series above can be rewritten as follows,

$$
\sum_{k=1}^{\infty} \sum_{n=1}^{\infty} \frac{1}{k^{4 m}+k^{4 m-2} n^{2}}=\frac{1}{2} \sum_{k=1}^{\infty}\left(-\frac{1}{k^{4 m}}+\frac{\pi \operatorname{coth}(k \pi)}{k^{4 m-1}}\right) .
$$

Since the right-hand side expression above is always positive and monotonically decreases, Cauchy Condensation Test can be applied. Therefore, below is the necessary and sufficient condition for it to converge.

$$
\sum_{k=0}^{\infty} 2^{k}\left(-\frac{1}{\left(2^{k}\right)^{4 m}}+\frac{\pi \operatorname{coth}\left(2^{k} \pi\right)}{\left(2^{k}\right)^{4 m-1}}\right) .
$$

This series can be divided as follows,

$$
(-1+\pi \operatorname{coth} \pi)+\sum_{k=1}^{\infty} 2^{k}\left(-\frac{1}{\left(2^{k}\right)^{4 m}}+\frac{\pi \operatorname{coth}\left(2^{k} \pi\right)}{\left(2^{k}\right)^{4 m-1}}\right) .
$$

The expressions in the first parentheses are constants and by applying the ratio test, we can see that the part of the infinite series converges if the following conditions are satisfied.

$$
\lim _{k \rightarrow \infty}\left|\frac{2^{k+1}\left(-\frac{1}{\left(2^{k+1}\right)^{4 m}}+\frac{\pi \operatorname{coth}\left(2^{k+1} \pi\right)}{\left(2^{k+1}\right)^{4 m-1}}\right)}{2^{k}\left(-\frac{1}{\left(2^{k}\right)^{4 m}}+\frac{\pi \operatorname{coth}\left(2^{k} \pi\right)}{\left(2^{k}\right)^{4 m-1}}\right)}\right|<1 .
$$


The value of the left-hand side expression is $4^{1-2 m}$, which is less than 1 . Thus, the series converges.

\section{Lemma 2.3.}

$$
\sum_{k=1}^{\infty} \sum_{n=1}^{\infty} \frac{1}{k^{4 m}+k^{4 m-2} n^{2}}=\frac{1}{2} \sum_{k=1}^{\infty} \sum_{n=1}^{\infty} \sum_{s=1}^{2 m-1} \frac{(-1)^{s+1}}{k^{4 m-2 s} n^{2 s}}
$$

Proof.

$$
\sum_{s=1}^{2 m-1} \frac{(-1)^{s+1}}{k^{4 m-2 s} n^{2 s}}=\sum_{s=1}^{2 m-1} \frac{1}{k^{4 m}}\left((-1)^{s+1} \frac{k^{2 s}}{n^{2 s}}\right) .
$$

It is well-known that for an integer $p$, the following equation is valid

$$
\begin{aligned}
\frac{\alpha^{p}-\beta^{p}}{\alpha-\beta} & =\alpha^{p-1}+\alpha^{p-2} \beta+\alpha^{p-3} \beta^{2}+\cdots+\beta^{p-1} \\
& =\sum_{s=1}^{p} \alpha^{p-s} \beta^{s-1} \\
& =\frac{\alpha^{p}}{\beta} \sum_{s=1}^{p}\left(\frac{\beta}{\alpha}\right)^{s} .
\end{aligned}
$$

One can divide both sides by $1 / \alpha^{p} \beta^{p}$ to get

$$
\frac{1}{\alpha-\beta}\left[\frac{1}{\beta^{p}}-\frac{1}{\alpha^{p}}\right]=\frac{1}{\beta^{p+1}} \sum_{s=1}^{p}\left(\frac{\beta}{\alpha}\right)^{s} .
$$

Now, let $\alpha=n^{2}$ and $\beta=-k^{2}$ and $p=2 m-1$. This gives the equation below

$$
\frac{1}{k^{4 m}+k^{4 m-2} n^{2}}+\frac{1}{k^{2} n^{4 m-2}+n^{4 m}}=\sum_{s=1}^{2 m-1} \frac{(-1)^{s+1}}{k^{4 m-2 s} n^{2 s}} .
$$

From the result of Lemma 2.2, we can say that the infinite sum of the left-hand side and each term of the left-hand side converge, so we obtain the following equality.

$$
\sum_{k=1}^{\infty} \sum_{n=1}^{\infty} \frac{1}{k^{4 m-2} n^{2}+k^{4 m}}+\sum_{k=1}^{\infty} \sum_{n=1}^{\infty} \frac{1}{n^{4 m}+k^{2} n^{4 m-2}}=\sum_{k=1}^{\infty} \sum_{n=1}^{\infty} \sum_{s=1}^{2 m-1} \frac{(-1)^{s+1}}{k^{4 m-2 s} n^{2 s}} .
$$

Since these dual series are positive term series and converge, we can rewrite them as follows.

$$
\sum_{k=1}^{\infty} \sum_{n=1}^{\infty} \frac{2}{k^{4 m-2} n^{2}+k^{4 m}}=\sum_{k=1}^{\infty} \sum_{n=1}^{\infty} \sum_{s=1}^{2 m-1} \frac{(-1)^{s+1}}{k^{4 m-2 s} n^{2 s}} .
$$

\section{Proof of the main theorem}

The following relation is well-known.

$$
\zeta(2 n)=(-1)^{n+1} \frac{B_{2 n}(2 \pi)^{2 n}}{2(2 n) !} .
$$


We can rewrite Theorem 1.1 with this equation as follows.

$$
\zeta(4 m-1)+2 \sum_{k=1}^{\infty} \frac{1}{k^{4 m-1}\left(e^{2 \pi k}-1\right)}=\frac{1}{\pi} \sum_{s=0}^{2 m}(-1)^{s+1} \zeta(4 m-2 s) \zeta(2 s) .
$$

The equation above can be proved by transforming the equation from Corollary 2.1 using Lemma 2.3 by the following process.

$$
\begin{aligned}
\sum_{k=1}^{\infty} \frac{\operatorname{coth}(\pi k)}{k^{4 m-1}} & =\frac{1}{\pi} \sum_{k=1}^{\infty} \frac{1}{k^{4 m}}+\frac{2}{\pi} \sum_{k=1}^{\infty} \sum_{n=1}^{\infty} \frac{1}{k^{4 m-2} n^{2}+k^{4 m}} \\
& =\frac{1}{\pi}\left(\sum_{k=1}^{\infty} \frac{1}{k^{4 m}}+\sum_{k=1}^{\infty} \sum_{n=1}^{\infty} \sum_{s=1}^{2 m-1} \frac{(-1)^{s+1}}{k^{4 m-2 s} n^{2 s}}\right) \\
& =\frac{1}{\pi}\left(\zeta(4 m)+\sum_{s=1}^{2 m-1}(-1)^{s+1} \zeta(4 m-2 s) \zeta(2 s)\right) \\
& =\frac{1}{\pi} \sum_{s=0}^{2 m}(-1)^{s+1} \zeta(4 m-2 s) \zeta(2 s) .
\end{aligned}
$$

\section{Appendix}

This appendix gives the following equations.

$$
\begin{aligned}
& \frac{1}{e^{2}-1}=\sum_{n=1}^{\infty} \frac{1}{(n \pi)^{2}+1}, \\
& \frac{\pi}{e^{\pi}-1}=2 \sum_{n=1}^{\infty}\left(\frac{1}{4 n^{2}+1}+\frac{(-1)^{n}}{4 n^{2}-1}\right) .
\end{aligned}
$$

These equations result from the following corollary.

\section{Corollary 4.0.1 (cf. [3, Exercise 1001, p. 149]).}

$$
\frac{x}{e^{x}-1}=-\frac{x}{2}+1+2 \sum_{n=1}^{\infty} \frac{1}{4\left(\frac{\pi}{x} n\right)^{2}+1} .
$$

Substituting $2, \pi$ for $x$ in Corollary 4.0.1, we get the following equations, respectively:

$$
\begin{aligned}
& \frac{1}{e^{2}-1}=\sum_{n=1}^{\infty} \frac{1}{(n \pi)^{2}+1}, \\
& \frac{\pi}{e^{\pi}-1}=-\frac{\pi}{2}+1+2 \sum_{n=1}^{\infty} \frac{1}{4 n^{2}+1} .
\end{aligned}
$$


The following equality can be obtained by computation in this manner:

$$
\begin{aligned}
2 \sum_{n=1}^{\infty} \frac{(-1)^{n+1}}{4 n^{2}-1} & =2 \lim _{k \rightarrow \infty} \sum_{n=1}^{k} \frac{(-1)^{n+1}}{4 n^{2}-1} \\
& =2 \lim _{k \rightarrow \infty} \sum_{n=1}^{k}\left(\frac{1}{2 n-1}-\frac{1}{2 n+1}\right) \cdot \frac{(-1)^{n+1}}{2} \\
& =\lim _{k \rightarrow \infty}\left(\left(\frac{1}{1}-\frac{1}{3}\right)-\left(\frac{1}{3}-\frac{1}{5}\right)+\left(\frac{1}{5}-\frac{1}{7}\right)-\cdots \pm\left(\frac{1}{2 k-1}-\frac{1}{2 k+1}\right)\right) \\
& =\lim _{k \rightarrow \infty}\left(2 \cdot\left(\frac{1}{1}-\frac{1}{3}+\frac{1}{5}-\frac{1}{7} \cdots \pm \frac{1}{2 k-1}\right)-1 \mp \frac{1}{2 k+1}\right) \\
& =\frac{\pi}{2}-1,
\end{aligned}
$$

which after substitution in $(* *)$ establishes equation (A2).

\section{Acknowledgements}

The authors are grateful to the Mathematics Department of Kaijo School for the fruitful discussions. The authors would like to thank the anonymous referees for their detailed and competent comments and suggestions.

\section{References}

[1] Glaisher, J. W. L. (1875). On a Class of Identical Relations in the Theory of Elliptic Functions. Philosophical Transactions of the Royal Society of London, 165, 489-518. Available online: http://www.jstor.org/stable/109157

[2] Vepštas, L. (2012). On Plouffe's Ramanujan identities. The Ramanujan Journal, 27(4), 387-408.

[3] Volkovyskii, L. I., Lunts, G. L., \& Aramanovich, I. G. (1965). A Collection of Problems on Complex Analysis, Dover Publications, Inc., New York 